\title{
O Abrigo como Fator de Risco ou Proteção: Avaliação Institucional e Indicadores de Qualidade
}

\author{
Foster Care as Risk or Protective Factors: Institutional Evaluation \\ and Quality Indicators
}

\author{
Alessandra Salina-Brandão* \& Lúcia Cavalcante de Albuquerque Williams \\ Universidade Federal de São Carlos
}

\begin{abstract}
Resumo
Partindo-se de estudos referentes ao impacto da organização dos abrigos sobre o desenvolvimento de crianças e adolescentes institucionalizados, o presente trabalho objetivou: investigar as estratégias utilizadas por profissionais ao avaliarem abrigos, bem como os indicadores de qualidade que orientam essa tarefa. Participaram do estudo nove avaliadores e cinco coordenadores de abrigos. A coleta de dados deu-se por entrevistas, questionários e análise documental, sendo analisados segundo o referencial teórico da Psicopatologia do Desenvolvimento, especificamente referente aos fatores de risco e proteção. Os avaliadores utilizaram, de forma e frequiência variada, a visita à entidade como estratégia avaliativa. Os indicadores de qualidade identificados, também variaram. Obteve-se baixa freqüência de relatos sobre práticas educativas dos monitores e cuidados da entidade para manutenção dos vínculos familiares.

Palavras-chave: Abrigos para crianças e adolescentes; Rede de proteção à criança e ao adolescente; Fatores de risco e proteção; Avaliação institucional.

Abstract

Based on studies relating the impact of foster care organizations on the development of institutionalized children and adolescents, the present paper conducted an investigation of the strategies used by professionals in charge of foster care evaluation in Brazil, as well as the quality indicators used for this task. Nine evaluators and five foster care coordinators took part in the study. Data collection involved interviews, questionnaires and document analysis, using a framework from Developmental Psychopathology, specifically in terms of its contribution on risk and protective factors. Evaluators used the institutional visit as a predominant assessment strategy, conducting it in different ways and frequency. The quality indicators found were also varied. A low frequency of reports related to the educational practice of frontline staff and institutional efforts in order to maintain family ties were observed as well.

Keywords: Foster care; Child and adolescent protection network; Risk and protective factors; Institutional assessment.
\end{abstract}

A medida de proteção denominada abrigo é descrita pelo Estatuto da Criança e do Adolescente ([ECA], 1990) como uma estratégia provisória e excepcional, utilizável apenas como forma de transição para a colocação de crianças e adolescentes em situação de risco em família substituta. O Estatuto define características para essas instituições, entre elas, a preservação da liberdade, o atendimento personalizado, em pequenas unidades e grupos, privilegiando-se ações descentralizadas e municipalizadas.

Estudos sobre essa temática indicam que o abrigo pode tanto facilitar o desenvolvimento das crianças e adolescentes abrigados quanto dificultar, dependendo da presença ou ausência de fatores de proteção na entidade (Ajdukovic \& Franz, 2005; Arpini, 2003; Crawford, 2006;

* Endereço para correspondência: Rua Paraná, 3, Apto. 39, Jardim Coralina, Bauru, SP, Brasil, CEP 17030-023. E-mail: alessandrasalina@hotmail.com
Freitas, 1997; Scott \& Davis, 2006; A. C. Siqueira \& Dell'Aglio, 2006; M. D. Siqueira, 1997; Taussig \& Culhane, 2005; Zeanah, Smyke, Koga, \& Carlson, 2005).

Os fatores de proteção dos abrigos sinalizados pela literatura consultada são: habilidade dos funcionários dos abrigos de reconhecer os objetivos da instituição (Ali, Silveira, \& Lunardelli, 2004); composição multidisciplinar da equipe de profissionais dos abrigos (Maricondi, 1997); habilidade dos funcionários de identificar a relação entre seu trabalho e dos demais funcionários da entidade (Ali et al., 2004); plano de trabalho do abrigo compatível com os princípios do ECA (1990); ausência de rotatividade dos profissionais da entidade (Maricondi, 1997; Shaw, 2006); cultura institucional que proporcione a educação continuada dos funcionários (Ali et al., 2004; Maricondi, 1997); habilidade dos profissionais para o trabalho em equipe (Ali et al., 2004); práticas diárias da instituição que proporcionem a organização da rotina dos abrigados (Ali et al., 2004; Carvalho, 1993); rotina 
da entidade que englobe a participação das crianças e adolescentes em atividades fora dos abrigos; estrutura física compatível com o número de abrigados (ECA, 1990); oferecimento de oportunidades para a profissionalização dos adolescentes abrigados (Carvalho, 1993); práticas educativas com objetos trazidos da casa das crianças/adolescentes; registros dos momentos vividos pelos abrigados na instituição (Prada, 2002; Weber, 2001); práticas que impeçam o desmembramento de irmãos (ECA, 1990); políticas que evitem a transferência de abrigados para outras entidades (ECA, 1990); práticas educativas positivas dos funcionários aos adolescentes abrigados (Prada, 2002; Prada \& Williams, 2007); ações de preservação dos vínculos familiares ou integração com a família substituta (Maricondi, 1997; Prada, 2002) e qualidade do espaço físico (Carvalho, 1993; ECA, 1990; Prada, 2002).

Além da forma da organização dos abrigos, um conjunto amplo de variáveis interfere na qualidade do serviço prestado pelas entidades, como, por exemplo, a atuação da rede de proteção à criança e ao adolescente. A rede de proteção é formada por diferentes órgãos públicos que se inter-relacionam (Carvalho, 1993; A. C. Siqueira \& Dell 'Aglio, 2006) e, uma de suas funções é a fiscalização dos abrigos. Segundo o artigo 95 do ECA (1990), cabe ao Judiciário, Ministério Público e Conselhos Tutelares (CT) a tarefa de fiscalização das entidades.

Alguns estudos internacionais e nacionais caracterizam o papel da rede de proteção à criança e adolescentes, junto aos abrigos. Um estudo, em Israel, teve o objetivo de acompanhar o bem-estar de crianças expostas à situações de risco (abusos ou negligência), após a decisão dos conselheiros tutelares de mantê-las ou tirá-las de suas casas. As crianças que ficaram em casas-lares tiveram um escore de qualidade de vida superior àquelas que ficaram em instituições do tipo convencional (DavidsonArad, Englechin-Segal, \& Wozner, 2003). Provavelmente, características de casas-lares, como presença da famíliasocial, número reduzido de crianças e espaço físico similar ao de uma casa convencional (Prada, 2002), tiveram um impacto positivo sobre o desenvolvimento das crianças, justificando a relação de dependência entre fatores de proteção e desenvolvimento adequado de crianças institucionalizadas ${ }^{1}$.

Um estudo, nos Estados Unidos, teve o objetivo de avaliar os efeitos de três características dos conselheiros tutelares na promoção do bem-estar de crianças que estavam em abrigos, sob a tutela do Estado (Ryan, Garnier, Zyphur, $\&$ Zhai, 2006). Especificamente, buscou-se avaliar se, a freqüência de visitas do conselheiro tutelar às crianças abrigadas, seu nível de formação e etnia comparada à etnia da criança visitada, favoreciam o cumprimento da estadia da criança e a reunificação com sua família. Ve-

${ }^{1} \mathrm{O}$ conceito de qualidade de vida foi definido pelos autores como a função efetiva da criança em 16 áreas obtidas a partir da combinação de quatro campos de atividade (psicológico, físico, social e cultural) e quatro modelos de ação (expressão, adaptação, integração e conservação). As autoras utilizaram um instrumento que abordou tais aspectos. rificou-se que, os abrigados que recebiam visitas mais freqüentes dos conselheiros cumpriam a estadia esperada nos abrigos; as crianças que eram acompanhadas por conselheiros com grau de mestrado ficavam em média 5,15 meses a menos nas entidades; e as crianças negras tiveram menor probabilidade de serem adotadas, independente da etnia do conselheiro (Ryan et al., 2006). Esse trabalho sugere que a formação continuada dos conselheiros tutelares por meio do mestrado pode ter contribuído para que os mesmos fossem mais eficientes para a colocação das crianças em famílias substitutas.

Dois estudos nacionais sinalizaram a dificuldade de viabilizar ações conjuntas entre os órgãos que compõem a rede de proteção. Um estudo realizado no Rio Grande do Sul, no Brasil, teve o objetivo de descrever os fatores de risco e de proteção da rede de atendimento a crianças e adolescentes vítimas de violência sexual. Entre os resultados obtidos, os autores ressaltaram que a falta de comunicação entre as instituições da rede de atendimento dificultou o cumprimento das medidas de proteção necessárias, aspecto evidenciado, por exemplo, pelos seguintes fatores: morosidade na articulação dos serviços oferecidos pela rede de apoio, fugas das crianças dos abrigos (e de casa) e falta de envolvimento da família nas intervenções realizadas (Habigzang, Azevedo, Koller, \& Machado, 2006).

Um segundo estudo realizado no Brasil teve o objetivo de compreender a aplicação das prerrogativas previstas no ECA (1990), no que tange ao direito à escolarização (Souza, Teixeira, \& Silva, 2003). Verificou-se que a relação entre a Escola e o CT investigado era marcada pela informalidade, não havia contato freqüente e sistematizado entre as duas instituições. A Escola não informava o CT a respeito dos casos de evasão escolar e o Conselho, por sua vez, não cobrava tal conduta. Não havia ações conjuntas entre Escola e CT (Souza et al., 2003).

Outro estudo brasileiro teve o objetivo de capacitar conselheiros tutelares para identificar os principais fatores de risco e de proteção que influenciam o desenvolvimento infantil por meio de um curso de capacitação. Os resultados do trabalho apontaram para um aumento na média de acertos dos participantes em todos os instrumentos de avaliação que foram aplicados antes e após o curso, indicando a eficácia do trabalho (Marcondelli, 2004). O trabalho indicou a inabilidade dos conselheiros em identificar à quais fatores de risco e proteção crianças e adolescentes são expostos, aspecto que também será investigado no presente estudo.

A partir da constatação de que os profissionais que atuam junto aos abrigos interferem no desenvolvimento das crianças e adolescentes, o presente trabalho busca responder a seguinte questão: como ocorre a atuação dos órgãos de proteção junto aos abrigos de crianças e adolescentes? Considerando a gama de atividades desenvolvidas por esses profissionais, selecionaram-se, para análise, ações relativas à fiscalização dos abrigos.

O presente trabalho caracteriza-se por um estudo de levantamento (Cozby, 2006) e teve os seguintes objeti- 
vos: (a) descrever as estratégias utilizadas pelos profissionais ao avaliarem os abrigos e (b) identificar quais indicadores de qualidade são considerados pelos profissionais quando avaliam essas entidades. Por indicadores de qualidade entendeu-se os itens sugeridos pelo ECA (1990) para funcionamento dos abrigos, bem como as sugestões da literatura da área já descritas neste teste.

\section{Método}

\section{Instrumentos}

Em um primeiro momento, foi realizado um estudo piloto, em uma cidade de pequeno porte do estado de São Paulo (aproximadamente 30 mil habitantes), com o objetivo de testar os instrumentos ${ }^{2}$ elaborados. Participaram dessa etapa oito avaliadores de abrigos e uma coordenadora de um dos abrigos.

$\mathrm{Na}$ seqüência, foi realizado o estudo final em um município de médio porte do centro oeste paulista, com aproximadamente 350 mil habitantes. Dos participantes, nove eram profissionais responsáveis pela avaliação dos abrigos e cinco eram coordenadores dessas entidades.

Os instrumentos utilizados para coleta de dados consistiram em:

Roteiro para Entrevista Individual com os Avaliadores dos Abrigos. Esse instrumento teve o objetivo de investigar se os profissionais responsáveis pela avaliação dos abrigos realizavam o monitoramento das entidades, como o faziam e com que freqüência.

Roteiro para Entrevista Individual com Coordenadores dos Abrigos. Esse instrumento teve o objetivo de levantar informações sobre: (a) a qualidade do serviço prestado pela entidade, ou seja, quais indicadores de qualidade, sugeridos pela literatura, estavam presentes nos abrigos; (b) a avaliação dos coordenadores referente ao funcionamento dos abrigos e às estratégias de monitoramento utilizadas pelos órgãos competentes.

Roteiro para Análise dos Relatórios Elaborados pelos Avaliadores dos Abrigos. Esse instrumento (Anexo A) consistiu em um roteiro para análise dos documentos que descreviam as ações dos avaliadores junto aos abrigos, especificamente a tarefa de avaliação. $\mathrm{O}$ instrumento abordou: aspectos observados pelos avaliadores dos abrigos, comportamentos dos avaliadores (por exemplo, se faziam perguntas e/ou orientações) e qualidade dos encaminhamentos. Para a confecção deste instrumento foi realizado um levantamento dos indicadores de qualidade dos abrigos referidos pelo ECA (1990) e dos trabalhos dos seguintes autores: Ali et al. (2004); Carvalho (1993); Maricondi (1997); Prada (2002); Prada e Williams (2007) e Weber (2001).

Questionário sobre Indicadores de Qualidade dos Abrigos para os Avaliadores das Entidades. Esse instru-

${ }^{2}$ Todos os instrumentos utilizados, tanto no estudo piloto quanto no estudo final, foram elaborados pela primeira autora deste trabalho. mento (Anexo B) buscou complementar os dados obtidos por meio dos instrumentos anteriores acrescentando-se detalhamentos às respostas afirmativas ou negativas.

\section{Procedimento de Coleta dos Dados}

Para a realização da presente pesquisa, obteve-se aprovação do Comitê de Ética. Em seguida, buscou-se o consentimento da presidente do Conselho Municipal dos Direitos da Criança e dos Adolescentes (CMDCA). Após sua aprovação, foram convidados a participar da pesquisa os profissionais responsáveis pela avaliação e coordenação dos abrigos, que após terem sido esclarecidos sobre os objetivos e os cuidados éticos que seriam tomados, aceitaram participar. As entrevistas ocorreram nas próprias instituições. A pesquisa documental foi realizada após anuência do Juiz da cidade. Os documentos acessados consistiam em registros das visitas de monitoramento às entidades de atendimento.

\section{Tratamento e Análise dos Dados}

As entrevistas foram transcritas e em seguida foram elaboradas categorias e subcategorias de informações. $\mathrm{O}$ referencial utilizado para análise dos dados foi o campo teórico da Psicopatologia do Desenvolvimento, especificamente o conteúdo sobre fatores de risco e proteção para o desenvolvimento infantil (Maia \& Williams, 2005).

\section{Resultados}

A Tabela 1, a seguir, apresenta os dados que caracterizam os profissionais entrevistados: os avaliadores dos abrigos e os coordenadores das entidades.

Dentre os nove avaliadores, havia cinco conselheiros tutelares do município, uma conselheira do CMDCA, um juiz da Vara da Infância e da Juventude, um promotor da cidade e uma integrante da Secretaria do Bem Estar Social (SEBES). Esta última tinha a função de monitorar os abrigos. A idade média dos participantes foi de 38 anos, variando de 28 a 56 anos, sete participantes eram do sexo feminino e dois do sexo masculino, sendo que o tempo médio de atuação dos participantes foi de 5 anos (variando de 0,3 a 15 anos).

Todos os abrigos possuíam coordenador. Em alguns abrigos, o coordenador exercia dupla função. No abrigo 1 , por exemplo, a coordenadora exercia também função de assistente social. Todos os coordenadores possuíam nível superior de escolaridade. A idade média dos participantes foi de 37,8 anos, variando entre 26 e 52 anos. O tempo médio de atuação dos profissionais nos abrigos foi de 2,8 anos, variando entre 0,6 e 9 anos.

\section{Estratégias Utilizadas para a Avaliação/Fiscalização dos Abrigos}

A apresentação dos dados a respeito das estratégias de monitoramento dos abrigos, foi organizada da seguinte maneira: em um primeiro momento foram descritas as situações que proporcionavam a avaliação dos abrigos e, 
Salina-Brandão, A. \& Williams, L. C. A. (2009). O Abrigo como Fator de Risco ou Proteção: Avaliação Institucional e Indicadores de Qualidade.

Tabela 1

Caracterização dos Participantes

\begin{tabular}{clcclc}
\hline Participantes & $\begin{array}{c}\text { Formação } \\
\text { Universitária }\end{array}$ & Sexo & Idade & Função & $\begin{array}{c}\text { Tempo de } \\
\text { atuação/anos }\end{array}$ \\
\hline A1 & Terapeuta Ocupacional & F & 37 & Conselheira Tutelar & 3 \\
A2 & Advocacia & F & 41 & Conselheira Tutelar & 3 \\
A3 & Serviço Social & F & 32 & Conselheira Tutelar & 3 \\
A4 & Serviço Social & F & 35 & Conselheira Tutelar & 1,9 \\
A5 & Serviço Social & F & 28 & Conselheira Tutelar & 3 \\
A6 & Serviço Social e Pedagogia & F & 55 & Conselheira Municipal da & \\
& & & & Secretaria de Assistência Social & 4 \\
A7 & Serviço Social & F & 43 & Integrante da SEBES & 0,3 \\
A8 & Advocacia & M & 30 & Promotor & 15 \\
A9 & Advocacia & M & 40 & Juiz & 12 \\
C1 & Serviço Social & F & 30 & Coordenadora Abrigo & 1,8 \\
C2 & Psicologia & F & 37 & Coordenadora Abrigo & 0,6 \\
C3 & Serviço Social & F & 26 & Coordenadora Abrigo & 2,1 \\
C4 & Administração & M & 44 & Coordenador Abrigo & 9 \\
C5 & Serviço Social & F & 52 & Coordenadora Abrigo & 0,6 \\
& & & & & \\
\hline
\end{tabular}

na seqüência, os comportamentos apresentados pelos avaliadores dos abrigos ao monitorar as entidades. As estratégias de avaliação foram apresentadas em duas categorias: estratégias formais e informais. As estratégias formais referem-se às ações de avaliação planejadas pelos avaliadores, que consistiam em visitas ou acessos aos documentos dos abrigos. As estratégias informais referem-se às ações de avaliação dos abrigos não plane- jadas, mas que proporcionam dados importantes para a apreciação do funcionamento das instituições. Dentre as estratégias informais, enquadram-se as conversas dos avaliadores com adolescentes que fogem das instituições e com funcionários dos abrigos, quando esses procuram o Conselho Tutelar.

A Tabela 2, a seguir, apresenta as situações de avaliação das entidades relatadas pelos avaliadores.

Tabela 2

Situações de Avaliação

\begin{tabular}{lll}
\hline \multicolumn{1}{c}{ Situações de avaliação } & Profissionais \\
\hline Periodicidade & Continuamente & A8, A7 \\
& Nas fiscalizações semestrais & A1, A2, A3, A5, A6, A9 \\
& Nas fiscalizações bimestrais & A8 A5 \\
Motivos & No acompanhamento de crianças que abrigou & A2, A6, A7 \\
& Diante de denúncias & A2 \\
& Diante de ligações telefônicas e comparecimento & A6 \\
& de funcionário do abrigo no Conselho Tutelar & A6 \\
& Diante da solicitação do abrigo de registro de funcionamento & Para acompanhar a execução do projeto da entidade bem como \\
& a utilização de verba pública &
\end{tabular}

A Tabela 2 ilustra que todos os profissionais consultados relataram realizar algum tipo de avaliação, exceto a avaliadora A4. As demais conselheiras que relataram avaliar os abrigos, disseram que fiscalizam essas entidades semestralmente. O avaliador A8 (promotor) relatou realizar visitas de avaliação das entidades continuamente. Em relação aos motivos apresentados para avaliar os abrigos, o item "acompanhar crianças que abrigou", foi apresentado apenas pelas avaliadoras A2 e A5 e o item "atender denúncias" foi relatado apenas pelos participantes A2, A6 e A7.

A Tabela 3, a seguir, apresenta as estratégias formais e informais utilizadas pelos avaliadores para monitorar os abrigos. 
Tabela 3

Estratégias de Avaliação

\begin{tabular}{ll}
\hline Estratégias formais & Profissionais \\
\hline$\quad$ Visitar com agendamento prévio & A1, A2, A3, A5, A7 \\
$\varangle \quad$ Visitar sem agendamento prévio & A1, A2, A3, A8, A6, A7 \\
$-\quad$ Visitar com uma equipe (CMDCA, Ministério Público e Conselho Tutelar). & A1, A2, A3, A5, A8, A6, A7, A9 \\
$-\quad$ Conversar com funcionários durante as visitas & A1, A8 \\
- Dar feedback aos funcionários durante as visitas & A1 \\
$>\quad$ Conversar com crianças e adolescentes abrigados durante as visitas & A3 \\
$\quad$ Pesquisar prontuários durante as visitas & A1, A2, A3, A5, A8 \\
Solicitar o comparecimento de funcionárias do abrigo ao CT & A2 \\
Ler processos e relatórios enviados pelo abrigo & A8, A7 \\
Perguntar se o abrigo precisa de ajuda & A8 \\
Reunir-se com a equipe do abrigo & A1, A8, A7 \\
Reunir-se com a diretoria do abrigo & A7 \\
Verificar a participação da entidade em treinamentos oferecidos pela SEBES & A7 \\
Estratégias informais & A2, A8 e A7 \\
Conversar com funcionários por telefone & A2 \\
Conversar com funcionários dos abrigos quando estes procuram pessoalmente o CT & A2 \\
Conversar com crianças e adolescentes abrigados quando fogem do abrigo. &
\end{tabular}

Os avaliadores apresentam treze estratégias utilizadas para avaliar abrigos. A ação "visitar" foi a mais citada. Essa estratégia compreende sete ações relacionadas com a visita de avaliação.

Em relação às estratégias informais utilizadas para avaliar as entidades, os participantes A2, A8, A7 relataram conversar ao telefone com os funcionários dos abrigos, como uma forma de acompanhamento do funcionamento da entidade. Apenas A2 indicou as estratégias: conversar com funcionários dos abrigos quando estes procuram pessoalmente o CT e conversar com crianças e adolescentes abrigados quando esses fogem do abrigo.

\section{Estratégias de Fiscalização dos Abrigos Obtidas em Pesquisa Documental}

Havia apenas 11 registros disponíveis e desses, quatro eram referentes a monitoramentos realizados de forma individual pelos avaliadores do abrigo, tendo destaque os registros disponibilizados pelo Poder Judiciário, os quais diziam respeito às visitas feitas exclusivamente por A9. Três registros referiam-se ao monitoramento feito por uma equipe formada pelo CT, CMDCA e SEBES, dois registros indicavam a parceria do CT e CMDCA na realização desta tarefa, um documento referia-se ao monitoramento realizado pelo $\mathrm{CT}$ em parceria com a SEBES e um dos documentos remeteu-se a uma avaliação feita apenas por membros do CMDCA. Cabe ressaltar que foram pesquisados os documentos referentes aos anos de 2003, 2004 e 2005, os quais correspondiam ao período no qual a maior parte dos avaliadores entrevistados estava em exercício, ou seja, os conselheiros tutelares.
Quanto às informações contidas nos documentos referentes às ações realizadas pelos fiscalizadores para monitorar os abrigos, dos 11 registros disponíveis, oito revelaram que os avaliadores orientavam os coordenadores do abrigo a lidar com dificuldades da instituição, dois referiam que os fiscalizadores faziam perguntas aos coordenadores dos abrigos e quatro faziam menção ao estabelecimento de prazo para a regularização da entidade, sendo três desses documentos expedidos por A9.

\section{Relatos de Indicadores de Qualidade}

$\mathrm{Na}$ Tabela 4, a seguir, encontram-se os indicadores de qualidade descritos pelos participantes nas entrevistas, observados durante a tarefa de monitoramento.

Observa na Tabela 4 que dos 27 indicadores de qualidade apontados no questionário, os entrevistados indicaram 25 itens, considerados importantes para orientar as avaliações dos abrigos, indicando individualmente uma média de 6,7 itens por entrevistado. O avaliador que mais citou itens foi A3 (nove indicações), seguido por A6 (oito itens). Os participantes relataram que observavam uma média de 20 itens em suas atividades de monitoramento. $\mathrm{O}$ participante que menos citou itens foi A5, apenas três.

Cabe ressaltar que $56 \%$ dos itens foram citados apenas uma vez nesse estudo, ou seja, apenas um dos oito avaliadores enumerou tal aspecto como importante para a sua avaliação. Os itens mais citados foram: higiene, oferecimento de atividades para as crianças e adolescentes e qualidade das dependências do abrigo, os quais foram mencionados por 4, 5 e 6 participantes, respectivamente. 
Salina-Brandão, A. \& Williams, L. C. A. (2009). O Abrigo como Fator de Risco ou Proteção: Avaliação Institucional e Indicadores de Qualidade.

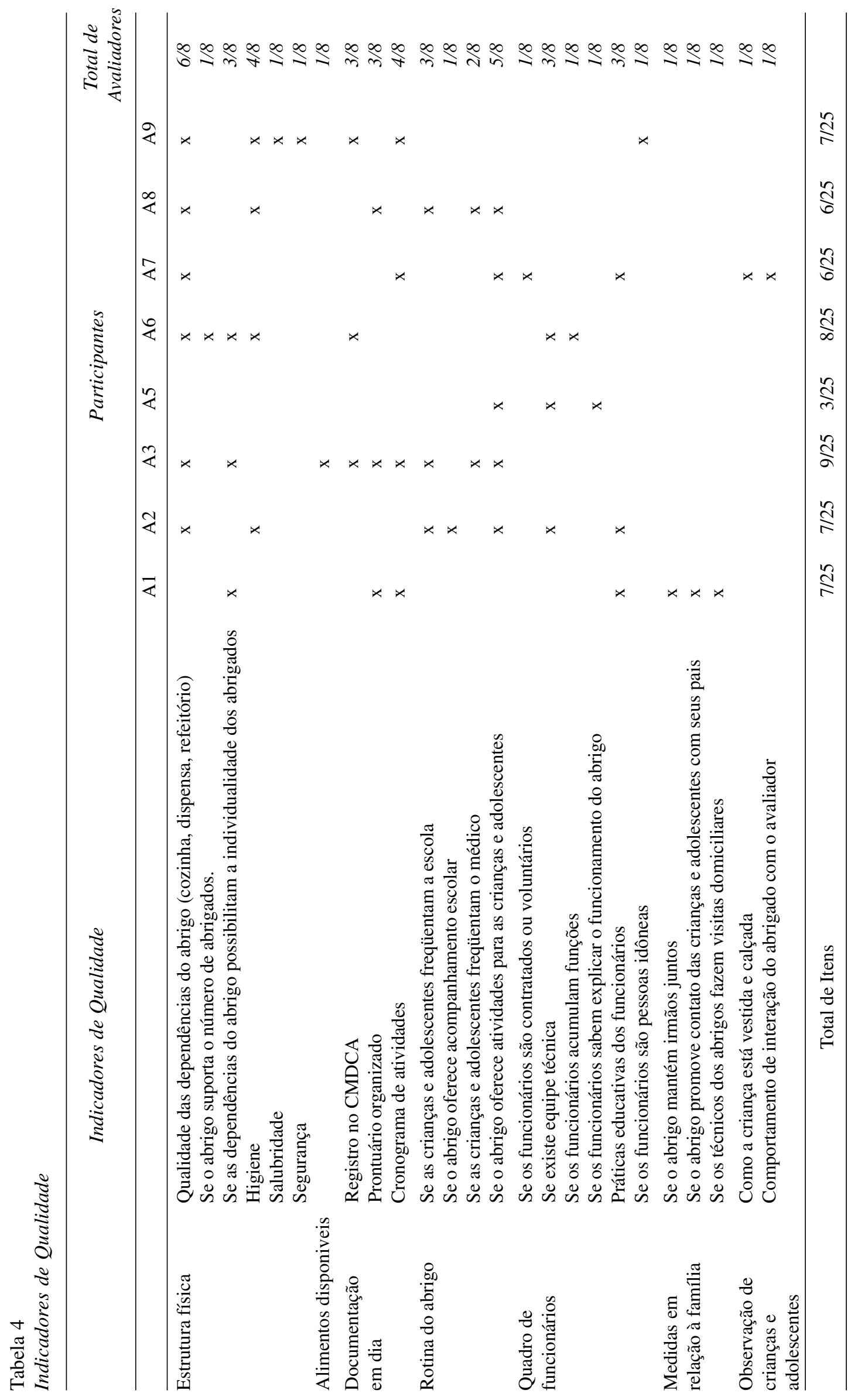




\section{Discussão}

O presente estudo teve o objetivo de investigar como é feita a fiscalização dos abrigos de crianças e adolescentes pelos órgãos competentes. Duas questões conduziram essa pesquisa: quais as estratégias utilizadas para a fiscalização dos abrigos e quais os indicadores de qualidade que norteiam as ações dos avaliadores.

Para analisar as estratégias relatadas pelos participantes, tornou-se importante discutir outro conjunto de dados apresentados pelos entrevistados: as situações nas quais ocorriam a fiscalização e a frequiência com a qual essa tarefa era realizada. Observou-se grande variabilidade de descrições de situações nas quais ocorriam a avaliação dos abrigos, denotando que os profissionais responsáveis pelo monitoramento das entidades não realizam essa tarefa de forma integrada, apesar de alguns avaliadores mencionarem que acompanhavam o funcionamento dos abrigos com demais colegas.

Habigzang et al. (2006) e Souza et al. (2003), encontraram resultados semelhantes. O primeiro trabalho sinalizou que a falta de comunicação entre as instituições da rede de atendimento dificultou o cumprimento das medidas de proteção necessárias às crianças e adolescentes vítimas de violência sexual e, o segundo artigo, indicou que a ausência de trabalhos integrados entre CT e escola prejudicava os cuidados relacionados à freqüência escolar de crianças e adolescentes.

Sobre a frequiência das avaliações, todos os avaliadores dos abrigos, exceto A4, responderam que monitoravam as entidades apenas semestralmente. Observa-se, também, grande diversidade de relato de estratégias utilizadas para avaliar o abrigo: os entrevistados citaram 16 estratégias diferentes, havendo maior concentração de respostas referentes às estratégias: visitar com/sem agendamento prévio e visitar com uma equipe de profissionais. Cabe ressaltar que o relato a respeito das estratégias de fiscalização por meio de uma equipe de profissionais é corroborado pelos registros presentes nos documentos analisados.

Outro aspecto importante proporcionado pela análise documental foi a comparação entre o relato das estratégias feito por meio da entrevista pelos participantes e descrições presentes nos documentos consultados. Apesar de não permitirem uma análise individualizada dos participantes, mas apenas do grupo que estava presente na avaliação, os registros denotam que as avaliações realizadas por A1, A6 e A7 apresentavam classes de comportamentos coerentes com as afirmações feitas nas entrevistas por essas participantes, como: dar feedback aos funcionários durante as visitas e pesquisar prontuários durante as visitas, aspectos citados por A1 em entrevista.

Possivelmente, algumas diferenças entre as estratégias utilizadas pelos avaliadores, também poderiam ser explicadas pelas particularidades de cada função. Por exemplo, apenas A7 descreveu que verificava a partici- pação dos coordenadores dos abrigos nos treinamentos oferecidos pela SEBES. Supõe-se que essa descrição isolada foi decorrente do fato dessa entrevistada ser funcionária da SEBES.

Outro aspecto relevante é a qualidade da estratégia utilizada, ou seja, em que medida determinada forma de abordagem ao abrigo pôde contribuir para a obtenção de dados que subsidiassem intervenções necessárias. A participante A2, por exemplo, descreveu uma estratégia que possivelmente não contribuía para o aprimoramento do abrigo: "telefonar para os abrigos para saber se estava tudo bem." Um dos aspectos que pode invalidar essa estratégia é o grau de fidedignidade do discurso do profissional que fornecerá as informações por telefone. Já a participante A3, mostrou habilidade para abordar as crianças e adolescentes a fim de avaliar as condições do abrigo, por exemplo, quando ela relatou que procurava sentar junto aos mesmos e iniciar a conversa de forma amigável.

Com o objetivo de sinalizar algumas habilidades que poderão ser desenvolvidas em capacitações futuras para avaliadores de abrigos, segue a descrição dos fatores de proteção ilustrados nos comportamentos dos participantes desse estudo, apesar dos mesmos aparecerem com baixa frequiência: visitar a entidade com alta freqüência (por exemplo, "quinzenalmente"); atender aos pedidos de ajuda dos abrigos (por exemplo, quando os funcionários solicitam a presença do CT em função de brigas das crianças e adolescentes abrigados); visitar com/sem agendamento prévio; visitar com uma equipe (por exemplo, CMDCA, ministério público e CT); conversar com funcionários e população abrigada durante as visitas; pesquisar prontuários; ler processos e relatórios enviados pelo abrigo; perguntar se o abrigo precisa de ajuda; reunir-se com a equipe do abrigo; verificar a participação dos funcionários das entidades em cursos oferecidos pela SEBES; solicitar informação por meio de técnicos do Judiciário e demais profissionais da rede de assistência aos abrigos e identificar indicadores de qualidade necessários para o funcionamento adequado das entidades.

Observa-se, também, déficits referentes ao comportamento de identificar alguns indicadores de qualidade específicos, segundo os autores Ali et al. (2004), Carvalho (1993), Maricondi (1997), Prada (2002; Prada \& Williams, 2007) e Weber (2001), além do ECA (1990). Tais indicadores dizem respeito à: freqüência com a qual as propostas da instituição são discutidas e revistas pelos funcionários; os critérios adotados pelo abrigo para selecionar os funcionários; se os coordenadores das entidades desempenham um papel ativo de liderança (por exemplo, fazendo reuniões); a rotatividade de profissionais; se os funcionários já receberam algum treino em relação ao ECA (1990); se existe coesão entre os funcionários; se os adolescentes participam de atividades que visem a profissionalização; se a instituição possibilita o uso dos objetos trazidos pelas crianças e adolescentes de sua casa; 
Salina-Brandão, A. \& Williams, L. C. A. (2009). O Abrigo como Fator de Risco ou Proteção: Avaliação Institucional e Indicadores de Qualidade.

se no abrigo existe o registro de momentos vividos na instituição, por exemplo, fotos; se a instituição mantém uma política de não desmembramento de grupos de irmãos; se a entidade evita, sempre que possível, a transferência de abrigados para outras entidades; se existe um programa de formação continuada para os funcionários e se há ações do abrigo em relação a preservação dos vínculos familiares e integração em família substituta.

Os déficits dos avaliadores dos abrigos em identificar fatores de risco importantes para o desenvolvimento de crianças e adolescentes abrigados vão ao encontro dos resultados obtidos por Marcondelli (2004), a qual realizou um estudo com Conselheiros Tutelares e constatou a inabilidade dos conselheiros em identificar esses aspectos. Esse resultado justifica a necessidade de se investir na formação continuada desses profissionais, pois, como indica os trabalhos de Davidson-Arad et al. (2003) e Ryan et al. (2006), a capacitação do avaliador do abrigo tem efeitos diretos na qualidade de vida dos abrigados.

Além da promoção de capacitação para os profissionais responsáveis pela avaliação, sugere-se que novas pesquisas sejam realizadas a respeito da atuação da rede de apoio à criança e ao adolescente junto aos abrigos. Um dos caminhos possíveis seria a adaptação de alguns aspectos presentes nesse estudo, como: alteração do procedimento de aplicação do Questionário de Indicadores de Qualidade do Abrigo, o qual possivelmente teria proporcionado outros dados se tivesse sido aplicado como um roteiro de entrevista ou se apresentasse a instrução de forma diferente, com a inclusão de perguntas a todos os avaliadores a respeito: da avaliação que faziam sobre o funcionamento de cada abrigo e da qualidade do serviço oferecido por cada instituição.

A viabilização do Questionário de Indicadores de Qualidade como um instrumento de avaliação dos abrigos pode contribuir para a realização dessa tarefa, principalmente para auxiliar os avaliadores a monitorar aspectos da entidade que são comumente negligenciados. Nesse sentido, sugere-se pesquisas futuras que investiguem a possível contribuição desse instrumento para a realização da tarefa de monitoramento e suas propriedades psicométricas.

Atreladas às ações acima citadas, sugerem-se políticas que previnam o abrigamento, como, por exemplo, o cuidado às famílias que se mostram incapazes de cuidar de seus filhos, como sugerido por Williams e Aiello (2004) quando discutem o conceito de empoderamento de famílias.

\section{Referências}

Ajdukovic, M., \& Franz, B. S. (2005). Behavioral and emotional problems of children by type of out-of-home care in Croatia. International Journal of Social Welfare, 14, 163-175.
Ali, N. S. A., Silveira, R. S. M., \& Lunardelli, M. C. F. (2004). Relato de experiência de estágio com monitores que trabalham em entidade que abriga menores em situação de risco. In E. Goulard Júnior, L. C. Câneo, \& M. C. F. Lunardelli (Eds.), Campo de estágio: Espaço de aprendizagem e diversidade (pp. 170-179). Bauru, SP: Joarte.

Arpini, D. M. (2003). Repensando a perspectiva institucional e a intervenção em abrigos para crianças e adolescentes. Psicologia: Ciência e Profissão, 23(1), 70-75. Retrieved September, 5, 2004, from SciELO database.

Carvalho, M. C. B. (1993). Trabalhando abrigos. São Paulo, SP: CBIA.

Cozby, P. C. (2006). Métodos de pesquisa em ciências do comportamento. São Paulo, SP: Atlas.

Crawford, M. (2006). Health of children in out-of-home care: Can we do better? Journal of Pediatrics and Child Health, 3, 77-78. Retrieved January 10, 2007, from Web of Science database.

Davidson-Arad, B., Englichin-Segal, D., \& Wozner, Y. (2003). Short-term follow-up children at risk: Comparison of the quality of life of children removed from home and children remaining at home. Child: Abuse \& Neglect, 7, 733-750. Retrieved January 10, 2007, from Web of Science database.

Estatuto da Criança e do Adolescente. (1990, 16 set.). Lei Federal no 8069/1990. Diário Oficial da União (Brasília, DF).

Freitas, M. J. (1997). Aspectos do desenvolvimento de um grupo de adolescentes albergados e suas implicações para uma intervenção psicoeducacional. Dissertação de Mestrado nãopublicada, Programa de Pós-Graduação em Educação, Universidade Estadual de Londrina, PR.

Habigzang, L. F., Azevedo, G. A., Koller, S. H., \& Machado, P. X. (2006). Fatores de risco e de proteção na rede de atendimento a crianças e adolescentes vítimas de violência sexual. Psicologia: Reflexão e Crítica, 19(3). Retrieved November 5, 2007, from SciELO database.

Maia, J. M. D., \& Williams, L. C. A. (2005). Fatores de risco e proteção ao desenvolvimento infantil: Uma revisão de área. Temas em Psicologia, 13(2), 91-103. Retrieved July 8, 2005, from SciELO database.

Marcondelli, J. (2004). Capacitação de conselheiros tutelares: Instruir para aprimorar. Dissertação de Mestrado nãopublicada, Programa de Pós-Graduação em Educação Especial, Universidade Federal de São Carlos, SP.

Maricondi, M. A. (1997). Falando de abrigo: Cinco anos de experiência do projeto casas de convivência. São Paulo, SP: Fundação Estadual do Bem Estar do Menor.

Prada, C. G. (2002). A família, o abrigo e o futuro: Análise de relatos de crianças que vivem em instituições. Dissertação de Mestrado não-publicada, Programa de Pós-Graduação em Psicologia da Infância e Adolescência, Universidade Federal do Paraná, Curitiba, PR.

Prada, C. G., \& Williams, L. C. A. (2007). Efeitos de um programa de práticas educativas para monitoras de um abrigo infantil. Revista Brasileira de Terapia Comportamental e Cognitiva, 9(1), 63-80.

Ryan, J. P., Garnier, P., Zyphur, M., \& Zhai, F. (2006). Investigating the effects of caseworker characteristics in child welfare. Children and Youth Services Review, 5, 721-736. Retrieved January 10, 2007, from Web of Science database.

Scott, L. D., \& Davis, L. E. (2006). Young, black, and male in foster care: Relationship of negative social contextual experiences to factors relevant to mental health service delivery. Journal of Adolescence, 29, 721-736. Retrieved January 10, 2007, from Web of Science database. 
Shaw, T. V. (2006). Reentry into the foster care system after reunification. Children and Youth Services Review, 28, 3751390. Retrieved January 10, 2007, from Web of Science database.

Siqueira, A. C., \& Dell'Aglio, D. D. (2006). O impacto da institucionalização na infância e adolescência: Uma revisão de literatura. Psicologia \& Sociedade, 18(1), 71-80.

Siqueira, M. D. (1997). A vida escorrendo pelo ralo: As alternativas de existência dos meninos de rua. Estudos de Psicologia, 2, 161-174. Retrieved September 5, 2004, from SciELO database.

Souza, M. P. R., Teixeira, D. C. S., \& Silva, M. C. Y. G. (2003, jan./jun.) Conselho tutelar: Um novo instrumento social contra o fracasso escolar? Estudos de Psicologia, 8(1), 71-82. Retrieved September 5, 2004, from SciELO database.

Taussig, H. N., \& Culhane, S. E. (2005). Foster care as an intervention for abuse and neglected a children. In K. A. Kendall-Tackett \& S. M. Giacomoni (Eds.), Child victimization: Maltreatment, bulling and dating violence, prevention and intervention (pp. 1-20). Kingston, NJ: Civic Research Institute.

Weber, L. N. D. (2001). A prevenção em instituições que abrigam crianças e a preparação e acompanhamento de pais adotivos [Resumo]. In Sociedade Brasileira de Psicologia (Ed.), Anais da XXXI Reunião Anual de Psicologia (pp. 6). Rio de Janeiro, RJ: Sociedade Brasileira de Psicologia.

Williams, L. C. A., \& Aiello, A. L. R. (2004). Empoderamento de famílias: O que vem a ser e como medir? In E. G. Mendes, M. A. Almeida, \& L. C. A. Williams (Eds.), Temas em educação especial: Avanços recentes (pp. 197-205). São Carlos, SP: Editora da Universidade Federal de São Carlos.

Zeanah, C. H., Smyke, A. T., Koga, S. F. \& Carlson, E. (2005). Attachment in institutionalized and community children in Romania. Child Development, 76, 10-15. Retrieved January 10, 2007, from Web of Science database. 
Salina-Brandão, A. \& Williams, L. C. A. (2009). O Abrigo como Fator de Risco ou Proteção: Avaliação Institucional e Indicadores de Qualidade.

\section{Anexo A}

Protocolo para Análise dos Relatórios Elaborados pelos Avaliadores dos Abrigos

\section{1) Aspectos observados pelos avaliadores dos abrigos} Características do abrigo

1) Se os funcionários da instituição descrevem as finalidades e objetivos que o abrigo pretende alcançar?

2) Se a instituição possui equipe com coordenador, monitor, assistente social, psicólogo, cozinheira e auxiliar de serviços gerais?

3) A freqüência com a qual as propostas da instituição são discutidas e revistas pelos funcionários.

4) Se os funcionários identificam a relação entre seu trabalho e dos demais funcionários do abrigo, por ex. se os monitores conhecem o papel da assistente social, etc...

5) Se os funcionários identificam o papel dos órgãos gestores do abrigo?

6) Os critérios adotados pelo abrigo para selecionar os funcionários?

7) Se os diferentes funcionários do abrigo têm clareza dos papéis a serem desenvolvidos? (descrevem suas ações de acordo com o sugerido pelo ECA e plano de trabalho da instituição).

8) Se os coordenadores dos abrigos desempenham um papel ativo e de liderança, por ex, fazendo reuniões com funcionários.

9) Se o plano de trabalho do abrigo é compatível com os princípios do ECA?

10) A rotatividade de profissionais.

11) Se os funcionários já receberam algum treino em relação ao Eca.

12) Se existe coesão entre os funcionários do abrigo, por exemplo, se existe colaboração entre os membros.

13) As práticas diárias da instituição, por ex, horário para a escola, refeição, número de refeições.

14) Se a estrutura do abrigo suporta o número de abrigados?

15) Se os abrigados participam de atividades fora do abrigo, como escolas, projetos comunitários, se freqüentam postos de saúde, igreja.

16) Se os adolescentes participam de atividades que visem a profissionalização.

17) Se o abrigo possibilita o uso dos objetos trazidos pelas crianças e adolescentes de sua casa.

18) Se no abrigo existe o registro de momentos vividos na instituição, por ex, fotos.

19) Se o abrigo mantém uma política de não desmembramento de grupos de irmãos.

20) Se o abrigo evita, sempre que possível, a transferência de abrigados para outras entidades.

21) Se existe um programa de formação continuada para os funcionários?

22) As práticas educativas dos monitores na interação com crianças e adolescentes do abrigo.

23) As ações do abrigo em relação a preservação dos vínculos familiares e integração em família substituta.

24) Os seguintes aspectos da estrutura física:

Iluminação/Arejamento

Acessibilidade arquitetônica

Higiene, salubridade e segurança

Disponibilidade de brinquedos

Disponibilidade de objetos e vestuários pessoais

25) Outros

2) Comportamentos dos avaliadores:
a) Fazem perguntas?
( ) $\mathrm{Sim}$
b) Orientam ações?
( ) $\mathrm{Sim}$
( ) Não
c) Outros
( ) Não
Em caso afirmativo:
São orientações: ( ) adequadas ( )inadequadas

Anexo B

Questionário sobre Indicadores de Qualidade dos Abrigos para os Avaliadores das Entidades

\begin{tabular}{ll}
\hline Dados pessoais: & Informações específicas: \\
Nome: & Data da aplicação \\
Idade & Horário de Início \\
Escolaridade & Horário de Término \\
Função/Tempo de atuação &
\end{tabular}

Identifique, conforme perguntas abaixo, os aspectos que procura observar nos abrigos quando os avalia:

Perguntas de: 1 a 25 Se sim: Detalhe dando exemplos Se não: Comente as dificuldades em relação

idem ao roteiro anterior

e indicando freqüência.

a avaliação deste aspecto. 